\title{
Engel, Nilpotent and Solvable BCI-algebras
}

\author{
Elahe Mohammadzadeh $^{(a)}$ and Rajab Ali Borzooei ${ }^{(b)}$
}

\begin{abstract}
In this paper, we define the concepts of Engel, nilpotent and solvable $B C I$-algebras and investigate some of their properties. Specially, we prove that any $B C K$-algebra is a 2-Engel. Then we define the center of a $B C I$-algebra and prove that in a nilpotent $B C I$-algebra $X$, each minimal closed ideal of $X$ is contained in the center of $X$. In addition, with some conditions, we show that every finite $B C I$-algebra is solvable. Finally, we investigate the relations among Engel, nilpotent and solvable $B C I(B C K)$-algebras.
\end{abstract}

\section{Introduction}

One of the most important concept in the study of groups is the notion of nilpotency [22]. Nilpotent groups arise in Galois theory, as well as in the classification of groups. Using Galois theory, certain problems in field theory can be reduced to group theory, which is, in some sense, simpler and better understood. Engel groups are certain generalized nilpotent groups which have received considerable attention in recent years $[4,5,15]$. In mathematics, more specifically in the field of group theory, a solvable group is a group that can be constructed from Abelian groups using extensions. And it is proved that every solvable group is nilpotent [22]. In 1966, Y. Imai and K. Iseki [7, 9], defined an algebra of type $(2,0)$, also known as $B C K$-algebra, as a generalization the notion of algebra sets with the subtraction set with the only a fundamental,

Key Words: BCI-algebra, Engel BCI-algebra, nilpotent BCI-algebra, solvable BCIalgebra.

2010 Mathematics Subject Classification: Primary 06F35, 08A05; Secondary 20F16, 20F18, 20F45.

Received: 30.01 .2018

Accepted: 08.05.2018 
non-nullary operation and the notion of implication algebra $[8,12]$ on the other hand. This notion is derived using two different methodologies, one of which is based on the set theory and the other on is from classical and nonclassical propositional calculi. In [18] Najafi, introduced the notion of pseudo commutator of two elements in a $B C K$-algebra. Then in [19], he used this notion to define a solvable $B C K$-algebra. Then, Najafi et.all in [20], gave a new definition of commutators and solvability in a $B C I$-algebras which have more properties. Moreover, they studied the notion of derived subalgebra, $X^{\prime}=[X, X]=\left\{\prod[x, y]: x, y \in\right\}$, which is a subalgebra but it is not an ideal of $X$ in general.

Now, in this paper, first we introduce a new definition for commutators of a $B C I$-algebra. Our motivation is that, a commutator of two elements in a $B C I$ algebra with the condition $0 * x=0$, is the same as commutator of two elements in a $B C K$-algebra, which this fact is not true with Najafie's definition. Then we generalized the notion of derived subalgebra $X^{\prime}=[X, X]=\langle\{[x, y]: x, y \in$ \}). In this case, $X^{\prime}$ is both a subalgebra and an ideal of $X$. Then, this new notion help to us to introduce a new definition for solvable $B C I$-algebras. We recall that in ring theory, an element $x$ in $(R,+, \cdot)$ is a nilpotent element if $x^{n}=0$, for some positive integer $n$. Also, an ideal $I$ of $R$ is called nilideal of $R$ if every element of $R$ is nilpotent. We can see that the concepts of nilpotency in a ring theory and group theory are not the same. In [6], Huang used the notion of nilpotency in ring theory to introduce the notion of nilpotency in BCI-algebra. Moreover, in [13] and [21], several results on these topics were obtained. Now, we used the notion of nilpotency in group theory to introduce the notion of nilpotency in $B C I$-algebra. This new definition of nilpotent $B C I$-algebras help to us to get a relation between nilpotent and solvabel $B C I$-algebras.

\section{Preliminary}

Bellow we recall some definitions and results that will be used in the rest of the paper.

An algebra $(X, *, 0)$ of type $(2,0)$ is called a $B C I$-algebra, if for any $x, y, z \in$ $X$, it satisfies the following axioms:

(BCI-1) $((x * y) *(x * z)) *(z * y)=0$,

$(\mathrm{BCI}-2)(x *(x * y)) * y=0$,

(BCI-3) $x * x=0$,

(BCI-4) $x * y=y * x=0$ implies $x=y$.

On any $B C I$-algebra $(X, *, 0)$, the natural order can be defined by putting $x \leq y$ if and only if $x * y=0$, for any $x, y \in X$. In a $B C I$-algebra $X$, if 
$0 * x=0$, for any $x \in X$, then it is called a $B C K$-algebra [7]. In a $B C K$ algebra $X$, for any $x, y, z \in X$, we have $(x * y) * z=(x * z) * y,(x * y) \leq x$, $x \leq y$ implies $x * z \leq y * z$ and $z * y \leq z * x, x * 0=x$. (See $[9,11,16]$ )

A $B C I$-algebra $X$ is said to be bounded if there exists an element $1 \in X$ such that $x \leq 1$, for any $x \in X$. For elements $x$ and $y$ of a $B C K$-algebra $X$, we denote $x \wedge y=y *(y * x)$ and $x \vee y=N(N x \wedge N y)$, where $N x=1 * x$. A $B C K$-algebra $X$ is said to be commutative if it satisfies $x \wedge y=y \wedge x$, for any $x, y \in X$. A nonempty subset $S$ of a $B C K$-algebra $X$ is called a subalgebra of $X$, if $x * y \in S$, whenever $x, y \in S$. Moreover, a nonempty subset $I$ of a $B C K$-algebra $X$ is called a $B C K$-ideal of $X$ if $0 \in I$ and if $x * y \in I$ and $y \in I$, then $x \in I$, for any $x, y \in X$. A $B C K$-algebra $X$ is called implicative, if for any $x, y \in X, x *(y * x)=x$ and $X$ is called a positive implicative, if for any $x, y, z \in X,(x * z) *(y * z)=(x * y) * z$. The number of elements of a $B C K$-algebra is called order of it. Let $(X, *, 0)$ and $(Y, ., 0)$ be two $B C K$ algebras. A mapping $f: X \longrightarrow Y$ is called a homomorphism from $X$ to $Y$, if for any $x, y \in X, f(x * y)=f(x) . f(y)$. Let $I$ be an ideal of a $B C K$-algebra $X$. Relation $\sim$ on $B C K$-algebra $X$ which is defined by $x \sim y$ if and only if $x * y, \quad y * x \in I$ is a congruence relation on $X$. Let $C_{x}$ denote the class of $x \in X$ and $X / I$ denote the set of all classes $C_{x}$, where $x \in X$. Then $X / I$ is a $B C K$-algebra with $C_{x} * C_{y}=C_{x * y}$ and $C_{x}=C_{y}$ if and only if $x \leq y$ and $X / I$ is called the quotient $B C K$-algebra of $X$ determined by $I$ (See [10]).

Theorem 2.1. [24] Let $X$ be a nonempty set. Then $X$ is a BCI-algebra if and only if there is a partial ordering $\leq$ on $X$ such that, for any $x, y, z \in X$, the following conditions hold:

(i) $(x * y) *(x * z) \leq(z * y)$,

(ii) $x *(x * y) \leq y$,

(iii) $x * y=0$ if and only if $x \leq y$.

Theorem 2.2. [24] Let $X$ be a BCI-algebra. Then for any $x, y, z \in X$, the following identities hold:

(i) $x \leq y$ implies $z * y \leq z * x$,

(ii) $x \leq y$ implies $x * z \leq y * z$,

(iii) $(x * y) * z=(x * z) * y$,

(iv) $x *(x *(x * y))=x * y$,

(v) $0 *(x * y)=(0 * x) *(0 * y)$,

(vi) $(x * y) *(z * y) \leq(x * z)$.

Let $x_{1}, x_{2}, x_{3}, \ldots, x_{n}$ be elements of $B C K$ algebra $X$. Then the element $\left(x_{1} \wedge x_{2}\right) *\left(x_{2} \wedge x_{1}\right)$ of $X$ is called the pseudo commutator of $x_{1}$ and $x_{2}$ of weight 2 and denoted by $\left[x_{1}, x_{2}\right]$. i.e., $\left[x_{1}, x_{2}\right]=\left(x_{1} \wedge x_{2}\right) *\left(x_{2} \wedge x_{1}\right)$. In general, the element $\left[x_{1}, x_{2}, \ldots \ldots, x_{n}\right]=\left[\left[x_{1}, \ldots ., x_{n-1}\right], x_{n}\right]$ is a commutator of weight 
$n \geq 2$, where $\left[x_{1}\right]=x_{1}$. A useful shorthand notation is $\left[x,_{n} y\right]=[x, y, \ldots, y]$ (See $[18]$ ).

Example 2.3. [18] Let $X=\{0,1,2,3,4\}$. If operation "*" on $X$ is defined by the following table:

\begin{tabular}{l|lllll}
$*$ & 0 & 1 & 2 & 3 & 4 \\
\hline 0 & 0 & 0 & 0 & 0 & 0 \\
1 & 1 & 0 & 1 & 0 & 0 \\
2 & 2 & 2 & 0 & 0 & 0 \\
3 & 3 & 3 & 3 & 0 & 0 \\
4 & 4 & 3 & 4 & 1 & 0
\end{tabular}

then $(X, *, 0)$ is a bounded positive implicative $B C K$-algebra with the largest element 4. We have $[2,4]=0 \neq 2=[4,2]$.

By Definition of $B C I$-algebra and Example 2.3, in the general case, $[x, y] \neq$ $[y, x]$, for any $x, y \in X$. Hence, the definition of commutator in $B C I$-algebras is not the same of definition of commutator in group theory. Thus the commutators defined in this paper, are essentially directed commutators. Now, we give some properties of commutators in $B C K$-algebras.

Lemma 2.4. [18] Let $X$ be a $B C K$-algebra. Then for any $x, y \in X$;

(i) if $x=y$, then $[x, y]=0$,

(ii) $[x, 0]=[0, x]=[x, x]=0$,

(iii) $[x, y] * x=0$,

(iv) $[x, y] * y=0$.

Let $x_{1}, x_{2}$ be elements of a $B C I$-algebra $X$. Then the element $\left(\left(x_{2} \wedge x_{1}\right) *\right.$ $\left.\left(x_{1} \wedge x_{2}\right)\right) *\left(0 *\left(x_{1} * x_{2}\right)\right)$ of $X$ is called a pseudo commutator of $x_{1}$ and $x_{2}$ of weight 2 and denoted by $\left[x_{1}, x_{2}\right]$, i.e. $\left[x_{1}, x_{2}\right]=\left(\left(x_{2} \wedge x_{1}\right) *\left(x_{1} \wedge x_{2}\right)\right) *$ $\left(0 *\left(x_{1} * x_{2}\right)\right)$. If $x_{1} * x_{2}=\left[x_{1}, x_{2}\right] *\left(x_{2} * x_{1}\right)$ or $x_{2} * x_{1}=\left[x_{2}, x_{1}\right] *\left(x_{1} * x_{2}\right)$, then $\left[x_{1}, x_{2}\right]$ is called the commutator of $x_{1}$ and $x_{2}$. If $x_{1}, x_{2}, \ldots, x_{n}(n \geq 2)$ be elements of $X$. We define $\left[x_{1}, x_{2}, \ldots, x_{n}\right]=\left[\left[x_{1}, x_{2}, \ldots, x_{n-1}\right], x_{n}\right]$ as a pseudo commutator of weight $n$. Let $X_{1}, \ldots, X_{n}$ be a non-empty subsets of $X$. Defined a commutator of $X_{1}$ and $X_{2}$ by

$$
\left[X_{1}, X_{2}\right]=\left\{\prod\left[x_{1}, x_{2}\right]: x_{1} \in X_{1} x_{2} \in X_{2}\right\} .
$$

More generally, for $n \geq 2$,

$$
\left[X_{1}, \ldots, X_{n}\right]=\left[\left[X_{1}, \ldots, X_{n-1}\right], X_{n}\right]
$$

$[X, X]$ is a product of a finite number of elements $[x, y]$ such that $x, y \in X$ and is called derived subalgebra of $X$ and is denoted by $X^{\prime} . X^{\prime}=[X, X]$, 
$X^{\prime \prime}=\left[X^{\prime}, X^{\prime}\right], X^{(n)}=\left[X^{(n-1)}, X^{(n-1)}\right]$. A BCI-algebra $X$ is called solvable if there exists $m \in \mathbb{N}$ such that $X^{(n)}=\{0\}$. (See [20]).

Notation. From now on, in this paper we let $X$ is a $B C I$-algebra, unless otherwise state.

\section{Engel $B C I$-algebras}

Now, in this section by using the notion of commutator of two elements in a $B C I$-algebra, we introduce and study the concept of Engel $B C I$-algebra and some of their properties.

Let $x, y \in X$. Define the commutator $[x, n y]$, for any $n \in \mathbb{N}$, by:

$[x, 0 y]=x, \quad[x, 1 y]=((y *(y * x)) *(x *(x * y))) *(0 *(y * x)), \quad\left[x,_{n} y\right]=\left[\left[x_{n_{n-1}} y\right], y\right]$.

Let us write $[x, \underbrace{y, \ldots, y}_{n}]$, for $\left[x,_{n} y\right]$. Therefore,

$$
\left[x,_{n} y\right]=[x, \underbrace{y, \ldots, y}_{n}]=[\ldots[[x, y], y], \ldots, y] .
$$

Clearly, in any $B C K$-algebra $X,[x, y]=(y *(y * x)) *(x *(x * y))$, for any $x, y \in X$.

Definition 3.1. A $B C I$-algebra $X$ is called an Engel BCI-algebra, if for each ordered pair $(x, y)$ of elements in $X$, there is a positive integer $n(x, y)$ such that $\left[x,_{n} y\right]=0$. Suppose $n=n(x, y)$ can be chosen independently of $x, y$. Then we say that $X$ is $n$-Engel BCI-algebra. Clearly every $n$-Engel $B C I$-algebra is Engel. Also, every finite $B C I$-algebra $X$ is $n$-Engel if and only if $X$ is Engel.

Example 3.2. Let $X=\{0,1, a, b, c\}$ and operation" * on $X$ is defined as follows:

\begin{tabular}{c|ccccc}
$*$ & 0 & 1 & $a$ & $b$ & $c$ \\
\hline 0 & 0 & 0 & $c$ & $c$ & $a$ \\
1 & 1 & 0 & $c$ & $c$ & $a$ \\
$a$ & $a$ & $a$ & 0 & 0 & $c$ \\
$b$ & $b$ & $a$ & 1 & 0 & $c$ \\
$c$ & $c$ & $c$ & $a$ & $a$ & 0
\end{tabular}

Then $(X, *, 0)$ is a BCI-algebra. Since for any $x, y \in X$ we have $[x, y]=0$, then $X$ is an 1-Engel BCI-algebra. 
Definition 3.3. [24] A $B C I$-algebra $X$ is called commutative if $x * y=0$ implies $x=y *(y * x)$, for any $x, y \in X$.

Theorem 3.4. [24] The following properties are equivalent on $X$ :

(i) $X$ is commutative,

(ii) $x *(x * y)=y *(y *(x *(x * y)))$, for any $x, y \in X$,

(iii) $(x *(x * y)) *(y *(y * x))=0 *(x * y)$, for any $x, y \in X$.

Theorem 3.5. $X$ is commutative if and only if $X$ is 1-Engel.

Proof. $\Longrightarrow$ Let $X$ be a commutative $B C I$ algebra. Then by Theorem 3.4, for any $x, y \in X,(y *(y * x)) *(x *(x * y))=(0 *(y * x))$, which implies that for any $x, y \in X,[x, y]=0$. Therefore, $X$ is 1-Engel.

$(\Longleftarrow)$ Let $X$ be an 1-Engel $B C I$-algebra and $x * y=0$. Then for any $x, y \in X, 0=[y, x]=((x *(x * y)) *(y *(y * x))) *(0 *(x * y))=x *(y *(y * x))$ and so $x \leq y *(y * x)$. Also clearly $y *(y * x) \leq x$. Therefore, $x * y=0$, implies $x=y *(y * x)$. Hence $X$ is commutative.

Lemma 3.6. For any $x, y \in X$, the following hold:

(i) $[x, y] * x \leq(0 * x)$,

(ii) $0 *[x, y]=0$,

(iii) $0 *[x, y]=[0 * x, 0 * y]$,

(iv) $x *[x, y] \leq x$,

(v) $[x, 0]=[0, x]=[x, x]=0$,

(vi) $[0 * x, y]=0$

Proof. (i) For any $x, y \in X$, we have

$$
\begin{aligned}
{[x, y] * x } & =((y *(y * x)) *(x *(x * y)) *(0 *(y * x))) * x \\
& \leq((x *(x *(x * y))) *(0 *(y * x))) * x \text { (by Theorems 2.2(ii) 2.1(ii)) } \\
& \leq((x * y) *(0 *(y * x))) * x \quad \text { (by Theorems 2.2(iv) and 2.1(ii)) } \\
& =((x * x) *(0 *(y * x))) * y \quad \text { (by Theorem 2.2(iii)) } \\
& =(0 *(0 *(y * x))) * y \\
& \leq(y * x) * y \quad \text { (by Theorems 2.2(ii) and 2.1(ii)) } \\
& =0 * x \quad \text { by Theorem 2.2(iii)). }
\end{aligned}
$$

(ii) By (i) and Theorem 2.2(vi), we have $0=([x, y] * x) *(0 * x) \leq[x, y] * 0=[x, y]$ and so by Theorem 2.1(iii), $0 *[x, y]=0$.

(iii) By (ii), $0 *[x, y]=0$ and so by definition of $[x, y]$ and Theorem 2.2(v), we 
have

$$
\begin{aligned}
& 0 *[x, y] \\
= & 0 *((y *(y * x)) *(x *(x * y)) *(0 *(y * x))) \\
= & (((0 * y) *((0 * y) *(0 * x))) *((0 * x) *((0 * x) *(0 * y))) \\
& *(0 *((0 * y) *(0 * x)))) \\
= & {[0 * x, 0 * y] . }
\end{aligned}
$$

(iv)

$$
\begin{aligned}
(x *[x, y]) * x & =(x * x) *[x, y] \quad(\text { by Theorem } 2.2(\text { iii })) \\
& =0 *[x, y] \quad(\text { by }(\text { BCI3) }) \\
& =0(\text { By part }(\text { ii }))
\end{aligned}
$$

Then by Theorem 2.1(iii), $x *[x, y] \leq x$.

(v) It is straightforward.

(vi) Let $x, y \in X$. Then

$$
\begin{aligned}
& {[0 * x, y] } \\
= & ((y *(y *(0 * x))) *((0 * x) *((0 * x) * y)) *(0 *(y *(0 * x)))) \\
\leq & ((0 * x) * y) *(0 *(y *(0 * x))) \quad(\text { by Theorems } 2.1(i i) 2.2(i v)) \\
\leq & ((0 * x) *(0 *(y *(0 * x)))) * y \quad(\text { by Theorem } 2.2(\text { iii })) \\
\leq & ((y *(0 * x)) * x) * y \\
= & ((y *(0 * x)) * y) * x \quad(\text { by Theorem } 2.2(\text { iii })) \\
= & ((y * y) *(0 * x)) * x \quad(\text { by Theorem } 2.2(i i i)) \\
= & (0 *(0 * x)) * x \\
= & (0 * x) *(0 * x) \quad(\text { by Theorem } 2.2(\text { iii })) \\
= & 0 .
\end{aligned}
$$

Example 3.7. Let $X=\{0, a, b\}$ and operation " * on $X$ is defined by:

\begin{tabular}{c|ccc}
$*$ & 0 & $a$ & $b$ \\
\hline 0 & 0 & $b$ & $a$ \\
$a$ & $a$ & 0 & $b$ \\
$b$ & $b$ & $a$ & 0
\end{tabular}

Then $X$ is a BCI-algebra. Since by Lemma 3.6, $[a, 0]=[0, a]=[a, a]=0$ and by $b * a=a, a * b=b$ we have $[a, b]=((b *(b * a)) *(a *(a * b))) *(0 *(b * a)=$ $((b * a) *(a * b)) *(0 * a)=b * b=0$. Similarly, $[b, a]=0$ and so for any $x, y \in X,[x, y]=0$. Therefore, $X$ is an 1-Engel BCI-algebra. 
Since any implicative $B C K$-algebra $X$ is a commutative $B C K$-algebra, then by Theorem 3.5, every implicative $B C K$ - algebra $X$ is 1-Engel.

Lemma 3.8. If $X$ is an $n$-Engel BCI-algebra, then $X$ is $(n+1)$-Engel.

Proof. Let $X$ be a $n$-Engel $B C I$-algebra. Then for any $x, y \in X,\left[x,{ }_{n} y\right]=0$. Hence, for any $x, y \in X$ we have $\left[x,_{n+1} y\right]=[[x, n y], y]=[0, y]=0$, which implies that $X$ is an $(n+1)$-Engel.

In the following Example we see that converse of Lemma 3.8, is not true in general.

Example 3.9. Let $X=[0,1]$ and operation " *" is defined by:

$$
x * y=\left\{\begin{array}{lc}
0, & x \leq y \\
x, & \text { otherwise }
\end{array}\right.
$$

Then $(X, *, 0)$ is a 2-Engel BCK-algebra. If for $x, y \in X, x \leq y$, then $[x, y]=$ 0 and $[y, x]=(x *(x * y)) *(y *(y * x))=x *(y *(y * x))=x$ which implies that $X$ is not 1-Engel. Now, since $[y, x, x]=[x, x]=0$, hence, $X$ is 2-Engel. Now, if $Z$ denote the cartesian product $X \times Y$ where $Y$ is as in Example 3.2. Then $(Z, *,(0,0))$ is a BCI-algebra, where operation " * "is defined by $\left(x_{1}, y_{1}\right) *\left(x_{2}, y_{2}\right)=\left(x_{1} * y_{1}, x_{2} * y_{2}\right)$, for any $x_{1}, x_{2} \in X$ and $y_{1}, y_{2} \in Y$ (See [24]). We show that $Z$ is not an 1-Engel BCI-algebra, but it is 2-Engel. First, we show that $\left[\left(x_{1}, y_{1}\right),\left(x_{2}, y_{2}\right)\right]=\left(\left[x_{1}, x_{2}\right],\left[y_{1}, y_{2}\right]\right)$. By the definition of commutator in a BCI-algebras and operation (*) on $Z$, we have:

$$
\begin{aligned}
& {\left[\left(x_{1}, y_{1}\right),\left(x_{2}, y_{2}\right)\right] } \\
= & \left(\left(\left(x_{2}, y_{2}\right) *\left(\left(x_{2}, y_{2}\right) *\left(x_{1}, y_{1}\right)\right)\right) *\left(\left(x_{1}, y_{1}\right) *\left(\left(x_{1}, y_{1}\right) *\left(x_{2}, y_{2}\right)\right)\right)\right) \\
& *\left((0,0) *\left(\left(x_{2}, y_{2}\right) *\left(x_{1}, y_{1}\right)\right)\right) \\
= & \left(\left(x_{2} *\left(x_{2} * x_{1}\right)\right),\left(y_{2} *\left(y_{2} * y_{1}\right)\right)\right) *\left(\left(x_{1} *\left(x_{1} * x_{2}\right)\right),\left(y_{1} *\left(y_{1} * y_{2}\right)\right)\right) \\
& *\left((0,0) *\left(x_{2} * x_{1}, y_{2} * y_{1}\right)\right) \\
= & \left(\left(\left(x_{2} *\left(x_{2} * x_{1}\right)\right) *\left(x_{1} *\left(x_{1} * x_{2}\right)\right) *\left(0 *\left(x_{2} * x_{1}\right)\right),\left(\left(\left(y_{2} *\left(y_{2} * y_{1}\right)\right)\right.\right.\right.\right. \\
& *\left(y_{1} *\left(y_{1} * y_{2}\right)\right) *\left(0 *\left(y_{2} * y_{1}\right)\right) \\
= & \left(\left[x_{1}, x_{2}\right],\left[y_{1}, y_{2}\right]\right)
\end{aligned}
$$

Then, $\left[\left(x_{1}, y_{1}\right),\left(x_{2}, y_{2}\right)\right]=\left(\left[x_{1}, x_{2}\right],\left[y_{1}, y_{2}\right]\right)$. Now, for some $x_{1}, x_{2} \in X$, $\left[x_{1}, x_{2}\right] \neq 0$ and by Example 3.2, $\left[y_{1}, y_{2}\right]=0$, for any $y_{1}, y_{2} \in Y$. Therefore, $\left[\left(x_{1}, y_{1}\right),\left(x_{2}, y_{2}\right)\right] \neq(0,0)$ which implies that $Z$ is not 1 -Engel. But $Z$ is 
2-Engel, since

$$
\begin{aligned}
{\left[\left(x_{1}, y_{1}\right)_{2}\left(x_{2}, y_{2}\right)\right] } & =\left[\left[\left(x_{1}, y_{1}\right),\left(x_{2}, y_{2}\right)\right],\left(x_{2}, y_{2}\right)\right] \\
& =\left[\left(\left[x_{1}, x_{2}\right],\left[y_{1}, y_{2}\right]\right),\left(x_{2}, y_{2}\right)\right] \\
& \left.=\left(\left[x_{1}, x_{2}\right], x_{2}\right],\left[\left[y_{1}, y_{2}\right], y_{2}\right]\right) \\
& =\left(\left[x_{1}, x_{2}\right],\left[y_{1}, y_{2}\right]\right) .
\end{aligned}
$$

Hence, $\left[\left(x_{1}, y_{1}\right)_{, 2}\left(x_{2}, y_{2}\right)\right]=\left(\left[x_{1}, 2 x_{2}\right],\left[y_{1,2} y_{2}\right]\right)$. Now, by Example 3.2, for any $x_{1}, x_{2} \in X,\left[x_{1}, x_{2}\right]=0$ and $\left[y_{1}, y_{2}\right]=0$ for any $y_{1}, y_{2} \in Y$. Then, $\left[\left(x_{1}, y_{1}\right)_{, 2}\left(x_{2}, y_{2}\right)\right]=\left(\left[x_{1}, 2 x_{2}\right],\left[y_{1,2} y_{2}\right]\right)=(0,0)$. Consequently, the converse of Lemma 3.8, is not true in general.

Note that, Example 3.9, is a 2-Engel BCI-algebra. Since $X$ is not 1-Engel, by Theorem 3.5, $X$ is not commutative. Therefore, Example 3.9, is an Engel $B C I$-algebra of class $n \neq 1$ which is not commutative. Therefore, the converse of Theorem 3.5, is not true for $n \neq 1$.

Theorem 3.10. Let $f$ be an isomorphism from $X$ to a BCI-algebra $Y$ and $n \in \mathbb{N}$. Then $X$ is an n-Engel BCI-algebra if and only if $Y$ is an n-Engel, too.

Proof. First, we show that $f\left(\left[a,_{n} b\right]\right)=\left[f(a)_{n} f(b)\right]$. For $n=1$;

$$
\begin{aligned}
f([a, b]) & =f(((b *(b * a)) *(a *(a * b))) *(0 *(b * a)) \\
& =(f(b *(b * a)) * f(a *(a * b))) *(f(0 *(b * a)) \\
& =((f(b) * f(b * a)) *(f(a) * f(a * b))) *(f(0) * f(b * a)) \\
& =((f(b) *(f(b) * f(a))) *(f(a) *(f(a) * f(b))) *(0 *(f(b) * f(a))) \\
& =[f(a), f(b)] .
\end{aligned}
$$

Thus, $f([a, b])=[f(a), f(b)]$. Now let $f\left(\left[a,{ }_{i} b\right]\right)=\left[f(a){ }_{i} f(b)\right]$, for $i \leq n$. We show that $f\left(\left[a,_{n+1} b\right]\right)=\left[f(a)_{, n+1} f(b)\right]$. By hypotheses of induction $\left[f(a)_{, n+1} f(b)\right]=\left[\left[f(a)_{n} f(b)\right], f(b)\right]=\left[f\left(\left[a,_{n} b\right]\right), f(b)\right]=f\left(\left[a,_{n+1} b\right]\right)$. Thus, $f\left(\left[a,{ }_{n} b\right]\right)=\left[f(a)_{n} f(b)\right]$. Consequently, let $X$ be $n$-Engel and $h, k$ are arbitrary elements of $Y$. Then there exists $a, b \in X$ such that $f(a)=h$ and $f(b)=k$. Therefore, $0=f(0)=f\left(\left[a,_{n} b\right]\right)=\left[f(a)_{n} f(b)\right]=\left[h,_{n} k\right]$ and so $Y$ is an $n$-Engel $B C I$-algebra. Conversely, let $Y$ be an $n$-Engel $B C I$-algebra. Then for any $a, b \in X, f\left(\left[a,_{n} b\right]\right)=\left[f(a)_{n} f(b)\right]=\left[h,_{n} k\right]=0=f(0)$ and so $\left[a,_{n} b\right]=0$. Hence, $X$ is an $n$-Engel.

Corollary 3.11. If $X$ is $n$-Engel, then any sub-algebras $X$ is n-Engel. Also if $I$ is a BCI-ideal of $X$, then $X / I$ is n-Engel, too. 
Theorem 3.12. The intersection of any two n-Engel sub-algebras of $X$, is $n$-Engel.

Proof. The proof is straightforward.

The above result can be generalized such that the intersection of any arbitrary family of subalgebras of an $n$-Engel $B C I$-algebra, is $n$-Engel. However, in general, the union of two $B C I$-subalgebras of a $B C I$-algebra, may not be a $B C I$-algebra(See [24]).

Remak: By Theorem 3.5, every 1-Engel $B C I$-algebra is a commutative $B C I$-algebra. Since commutative $B C I$-algebras form a variety, then 1-Engel $B C I$-algebras form a variety.

By the following example we show that $n$ - Engel $B C I$-algebras $(n \neq 1)$ do not form a variety.

Example 3.13. Consider the Wronskis algebra $(X, *, 0)$, where $X=N \cup A \cup B$ in which $N$ is the set of non-negative integers, $A=\left\{a_{n}: n \in N\right\}$ and $B=\left\{b_{n}: n \in N\right\}$ and each pair of the sets $N, A, B$ are disjoint. We define a binary operation " * " on $X$, for any $m, n \in N$, as follows:

$m * n=\max \{0, m-n\}$,

$m * a_{n}=m * b_{n}=0$,

$a_{m} * n=a_{m+n}$

$b_{m} * n=b_{m+n}$,

$a_{m} * a_{n}=b_{m} * b_{n}=n * m$,

$a_{m} * b_{n}=b_{m} * a_{n}=(n+1) * m$,

Then $(X, *, 0)$ is a BCI-algebra with BCI-ordering

$$
\begin{aligned}
& 0 \leq 1 \leq 2 \leq \ldots \leq a_{2} \leq a_{1} \leq a_{0} \\
& 0 \leq 1 \leq 2 \leq \ldots \leq b_{2} \leq b_{1} \leq b_{0}
\end{aligned}
$$

By ordinary calculations we see that $[x, y] \in\{0,1,2\}$. Thus, for any $x, y \in X$ we have $[[x, y], y]=[t, z]$ in which $t \in\{0,1,2\}$. Now since $N, N \cup A$ and $N \cup B$ are commutative. Then $[[x, y], y]=[t, y]=0$. Therefore, $X$ is a 2-Engel BCI-algebra.

The collection $\{N, A, B\}$ determines a partition of $X$. Thus we have a congruence relation $\theta$ on $X$, where $x \sim_{\theta} y$ if and only if both of $x$ and $y$ are in one of the tree sets $N, A$ and $B$. We obtain a quotient algebra $(X / \theta, *, N)$, where $X / \theta=\{N, A, B\}$. Now, it is easy to see that the "* multiplication table on $X / \theta$ is

\begin{tabular}{l|lll}
$*$ & $N$ & $A$ & $B$ \\
\hline$N$ & $N$ & $N$ & $N$ \\
$A$ & $A$ & $N$ & $N$ \\
$B$ & $B$ & $N$ & $N$
\end{tabular}


Since $A * B=B * A=N$ and $A \neq B$, we get $X / \theta$ is not a $B C I$-algebra. The mapping $f: X \longrightarrow X / \theta, x \longrightarrow \theta_{x}$ is an BCI-epimorphism. However, $X / \theta=f(X)$ is not a BCI-algebra. Thus, homomorphic image of an n-Engel $B C I$-algebra $X$ is not an n-Engel BCI-algebra.

Theorem 3.14. Let $I$ be an ideal of $X$ and $n, m \in \mathbb{N}$. If $I$ is an $m$-Engel and $X / I$ is $n$-Engel, then $X$ is a $(n+m)$-Engel.

Proof. Let $f$ be the natural homomorphism from $X$ onto $X / I$. First, we show that, for any $i \in \mathbb{N},\left[C_{x}, i C_{y}\right]=C_{[x, i y]}$. For $i=1$, by $C_{x} * C_{y}=C_{x * y}$, for any $C_{x} * C_{y} \in X / I$, we have

$$
\begin{aligned}
& {\left[C_{x}, C_{y}\right] } \\
= & \left(\left(C_{y} *\left(C_{y} * C_{x}\right)\right) *\left(C_{x} *\left(C_{x} * C_{y}\right)\right)\right) *\left(0 *\left(C_{0} *\left(C_{y} * C_{x}\right)\right)\right) \\
= & \left(C_{(y *(y * x)} * C_{(x *(x * y)}\right) *\left(0 * C_{0 *(y * x)}\right) \\
= & C_{[x, y]} .
\end{aligned}
$$

Therefore, $\left[C_{x}, C_{y}\right]=C_{[x, y]}$. Now, let it is true for $i<n$. We show that $\left.\left[C_{x, n} C_{y}\right]=C_{[x, n} y\right]$. By hypotheses of induction

$$
\begin{aligned}
& {\left[C_{x, n} C_{y}\right] } \\
= & {\left.\left[\left[C_{x, n-1} C_{y}\right], C_{y}\right]=\left[C_{[x, n-1} y\right], C_{y}\right] } \\
= & \left.\left.\left.\left(\left(C_{y} *\left(C_{y} * C_{[x, n-1} y\right]\right)\right) *\left(C_{[x, n-1} y\right] *\left(C_{[x, n-1} y\right] * C_{y}\right)\right)\right) \\
& *\left(C_{0} *\left(C_{y} * C_{[x, n-1}\right)\right) \\
= & \left.\left(C_{\left(y *\left(y *\left[x,{ }_{n-1} y\right]\right)\right.} * C_{\left(\left[x,{ }_{n-1} y\right] *([x, n-1\right.} y\right] * y\right) \\
= & \left.\left.C_{[[x, n-1} y\right], y\right] \\
= & \left.C_{[x, n} y\right]
\end{aligned}
$$

Now, $X / I$ is $n$-Engel. Hence for any $\left.C_{x}, C_{y} \in X / I, C_{0}=\left[C_{x, n} C_{y}\right]=C_{[x, n} y\right]$ and so $0 \sim\left[x,_{n} y\right]$, which implies that $\left[x,_{n} y\right] \in I$. Then, for any $x, y \in X$, since $\left[x,_{n} y\right] \in I$ and $I$ is $m$-Engel, we have $\left[\left[x_{n} y\right]_{m} y\right]=0$. Therefore, $X$ is $(n+m)$-Engel.

Theorem 3.15. Every BCI-algebra of order less than 5, is Engel.

Proof. If $X$ is a $B C I$-algebra of order 1, then It is clear that, $X$ is 1-Engel. Moreover, there is a unique proper $B C I$-algebra of order two as follows:

\begin{tabular}{l|ll}
$*$ & 0 & 1 \\
\hline 0 & 0 & 0 \\
1 & 1 & 0
\end{tabular}


By Lemma 3.6, $[x, y]=0$ for any $x, y \in X$ and so $X$ is 1-Engel. Also, there are two proper $B C I$-algebra $X$ of order three, with the following operations.

\begin{tabular}{c|ccc}
$*$ & 0 & $\mathrm{a}$ & $\mathrm{b}$ \\
\hline 0 & 0 & $\mathrm{~b}$ & $\mathrm{a}$ \\
$\mathrm{a}$ & $\mathrm{a}$ & 0 & $\mathrm{~b}$ \\
$\mathrm{~b}$ & $\mathrm{~b}$ & $\mathrm{a}$ & 0
\end{tabular}

\begin{tabular}{c|ccc}
$*$ & 0 & $\mathrm{a}$ & $\mathrm{b}$ \\
\hline 0 & 0 & 0 & $\mathrm{~b}$ \\
$\mathrm{a}$ & $\mathrm{a}$ & 0 & $\mathrm{~b}$ \\
$\mathrm{~b}$ & $\mathrm{~b}$ & $\mathrm{~b}$ & 0
\end{tabular}

Then it is easy to see that $[a, b]=0=[b, a]$ and so $[x, y]=0$, for any $x, y \in X$. Hence, $X$ is 1-Engel.

Also, there are 8 proper $B C I$-algebra of order four (see [24]). One of them is $X=\{0, a, b, c\}$ with the following operation:

\begin{tabular}{c|cccc}
$*$ & 0 & $\mathrm{a}$ & $\mathrm{b}$ & $\mathrm{c}$ \\
\hline 0 & 0 & $\mathrm{a}$ & $\mathrm{b}$ & $\mathrm{c}$ \\
$\mathrm{a}$ & $\mathrm{a}$ & 0 & $\mathrm{c}$ & $\mathrm{b}$ \\
$\mathrm{b}$ & $\mathrm{b}$ & $\mathrm{c}$ & 0 & $\mathrm{a}$ \\
$\mathrm{c}$ & $\mathrm{c}$ & $\mathrm{b}$ & $\mathrm{a}$ & 0
\end{tabular}

Clearly $I=\{0, a\}$ is an 1-Engel ideal of $X$. Moreover, $X / I=\left\{I_{0}=I_{a}, I_{b}, I_{c}\right\}$ is a $B C I$-algebra of order 3 and so it is 1-Engel too. Therefore, by Theorem $3.14, X$ is 2-Engel. Similarly, for any $B C I$-algebra of order 4 , there is an ideal $I$ of order 2 or 3 (See [24]) that makes $X / I$ a $B C I$-algebra of order 3 or 2 , respectively, and so $X / I$ is 1 -Engel. Then, by Theorem 3.14, $X$ is 2-Engel. Hence, we see that all $B C I$-algebras of order less than 5 are Engel.

Definition 3.16. [18] An ideal $I$ of $X$ is called a closed ideal, if it is also a subalgebra of $X$. In this case it is denoted by $I \unlhd^{c} X$.

Theorem 3.17. [24] If $H$ is a subalgebra of $X$ and $K$ is a closed ideal of $X$ ,then $H K / K \cong H /(H \cap K)$.

Theorem 3.18. Let $N$ be an n-Engel subalgebra of $X$ and $M$ be a closed $m$-Engel ideal of $X$. Then $M N$ is $m+n$-Engel.

Proof. Clearly $N \cap M$ is an ideal of $N$ and so $N /(N \cap M)$ is a $B C I$-algebra. Also, by Theorem 3.10, $N /(N \cap M)$ is $n$-Engel (by canonical map $f: N \longrightarrow$ $N /(N \cap M))$. Since by Theorem 3.17, $N M / M \cong N /(M \cap N)$, so by Theorem 3.14, $N M$ is an $n+m$-Engel $B C I$-algebra.

Remark. Let $I$ be a closed ideal of $X$ and $x \in I$. Since $I$ is both ideal and subalgebra of $X$, then $0 * x \in I$. 
Theorem 3.19. [24] Suppose that $A$ and $B$ are ideals of a BCI-algebra $Y$ and let $A B=\bigcup_{a \in A} B_{a}$, where $B_{a}$ is an equivalence class in $Y / B$. If $B$ is closed, then $A B=A+B$, where $A+B=\langle A \cup B\rangle$.

Theorem 3.20. Every finite BCI-algebra has a unique maximal Engel closed ideal.

Proof. Clearly, $\{0\}$ is an Engel closed ideal of $X$. If $\{0\}$ is maximal, then the proof is complete, otherwise there is an Engel closed ideal $N$ of $X$ such that $\{0\} \subset N \subseteq X$. If $N$ is maximal, the proof is complete, otherwise there is an Engel closed ideal $M_{1}$ of $X$ such that $\{0\} \subset N \subset M_{1} \subseteq X$. Continuing this process, we have $\{0\} \subset N \subset M_{1} \subset M_{2} \subset \ldots \subset X$. Since $X$ is finite, there is an Engel closed ideal $M_{n}$ of $X$ such that $X=M_{n}$. Also, if there are two Engel closed ideals $H$ and $K$ of $X$, then by Theorems 3.18 and $3.19, H K$ is an Engel closed ideal of $X$. Since $H$ and $K$ are maximal ideals of $X$, then $H=H K=K$. Thus there is a unique maximal Engel closed ideal of $X$.

Definition 3.21. Let $y \in X$ be a fixed element of $X, Z_{(0, y)}(X)=\{0\}$ and $Z_{(n, y)}(X)=\left\langle\left\{x \mid\left[x,_{n} y\right]=0\right\}\right\rangle$, for any $n \geq 1$. Then for any $y \in X$, the sequence of ideals $Z_{(0, y)}(X) \subseteq Z_{(1, y)}(X) \ldots \subseteq Z_{(n, y)}(X)$ is called the upper central series of $X$.

Theorem 3.22. Let $n \in \mathbb{N}$. Then $X$ is $n$-Engel if and only if for any $y \in X$, $Z_{(n, y)}(X)=X$.

Proof. ( $\Longrightarrow)$ Let $X$ be a $n$-Engel $B C I$-algebra and $z \in X$. Then for any $y \in X,[z, n, y]=0$ which implies that $z \in Z_{(n, y)}(X)$.

$(\Longleftarrow)$ Let for any $y \in X, Z_{(n, y)}(X)=X$ and $y, z$ be arbitrary elements of $X$. Therefore $y, z \in X=Z_{(n, y)}(X)$ and so $[z, n y]=0$ which implies that $X$ is $n$-Engel.

We have only one $B C K$-algebra of order one, that is, $X=\{0\}$ and so is Engel. Also, there is a unique $B C K$-algebra of order two, that is, $X=\{0,1\}$ with the following operation.

\begin{tabular}{l|ll}
$*$ & 0 & 1 \\
\hline 0 & 0 & 0 \\
1 & 1 & 0
\end{tabular}

By Lemma 2.4, $X$ is 1-Engel. Thus, all $B C K$-algebras with order less than 3, are Engel.

Theorem 3.23. Every BCK-algebra is 2-Engel. 
Proof. Let $X$ be a $B C K$-algebra and $A=[x, y]$, where $x, y \in X$. Then,

$$
\begin{aligned}
{\left[x_{2} y\right] } & =[[x, y], y] \\
& =[A, y] \\
& =(y *(y * A)) *(A *(A * y)) \\
& \leq(y *(A *(A * y))) *(y * A)(\text { by Theorem } 2.2(i i i)) \\
& \leq A *(A *(A * y)) \quad(\text { by }(B C I-1) \text { and Theorem } 2.1) \\
& \leq A * y \quad(\text { by Theorem } 2.2(i v)) \\
& =[x, y * y \\
& =0(\text { by Lemma } 2.4) .
\end{aligned}
$$

Therefore, $X$ is a 2-Engel $B C K$-algebra.

Recall that, the set $B$ of all positive elements of $X$ is called the $B C K$-part of $X[16]$.

Corollary 3.24. The BCK-part of $X$ is 2-Engel.

\section{Nilpotent $B C I$-algebras}

In this section the concept of a nilpotent $B C I$-algebra is introduced. This help us to get a $B C I$-algebra analog of nilpotent groups. Moreover, we obtain some main results of nilpotent groups on $B C I$-algebras.

Definition 4.1. Let $Z_{0}(X)=0, Z_{n}(X)=\left\langle\left\{x \mid\left[x, y_{1}, \ldots, y_{n}\right]=0\right.\right.$, for any $\left.\left.y_{1}, \ldots, y_{n} \in X\right\}\right\rangle$ for any $n \geq 1$. Clearly, by Lemma 3.6, $Z_{n}(X)$ is a closed ideal of $X$. The sequence of ideals $Z_{0}(X) \subseteq Z_{1}(X) \ldots \subseteq Z_{n}(X)$ is called the upper central series of $X$. Its $i$-th term $Z_{i}(X)$ is called the $\mathrm{i}$-th center of $X$. Now, $X$ is called nilpotent, if there exists $n \in \mathbb{N}$, such that $Z_{n}(X)=X$. The smallest such integer is called the class of $X$

By Theorem 3.4, it is easy to see that:

Theorem 4.2. $X$ is a commutative if and only if it is nilpotent of class at most 1.

Proof. ( $\Longrightarrow$ ) Let $X$ be a commutative. By Theorem 3.4, it is easy to see that it is nilpotent of class at most 1 .

$(\Longleftarrow)$ Let $X$ be a nilpotent of class at most 1 and $x * y=0$. Then for any $x, y \in X, 0=[y, x]=((x *(x * y)) *(y *(y * x))) *(0 *(x * y))=x *(y *(y * x))$ and so $x \leq y *(y * x)$. Also clearly $y *(y * x) \leq x$. Hence if $x * y=0$, then $x=y *(y * x)$. Therefore $X$ is commutative. 
Lemma 4.3. Let $i>0$. Then $\left[Z_{i}(X), X\right] \subseteq Z_{i-1}(X)$.

Proof. Let $z=[x, y]$, where $x \in Z_{i}$ and $y \in X$. Then for any $y_{1}, \ldots, y_{i-1} \in X$, $0=\left(\left[[x, y], y_{1}, \ldots, y_{i-1}\right]\right)=0$. Hence, $z \in Z_{i-1}(X)$.

Theorem 4.4. Let $f$ be an isomorphism from $X$ to $B C I$-algebra $Y$ and $n \in \mathbb{N}$. Then $X$ is nilpotent if and only if $Y$ is nilpotent.

Proof. First, we show that $f\left(\left[a, b_{1}, \ldots, b_{n}\right]\right)=\left[f(a), f\left(b_{1}\right), \ldots, f\left(b_{n}\right)\right]$ where $a, b_{1}, \ldots b_{n} \in X$. For $n=1$, since $f$ is homomorphism we have

$$
\begin{aligned}
f([a, b]) & =f(((b *(b * a)) *(a *(a * b))) *(0 *(b * a)))) \\
& =f(b *(b * a)) * f(a *(a * b)) * f(0 *(b * a)) \\
& =(f(b) * f(b * a)) *(f(a) * f(a * b)) *(f(0) * f(b * a)) \\
& =(f(b) *(f(b) * f(a))) *(f(a) *(f(a) * f(b)) *((0 *(f(b) * f(a)))) \\
& =[f(a), f(b)] .
\end{aligned}
$$

Thus, $f([a, b])=[f(a), f(b)]$. Now, let $f\left(\left[a, b_{1}, \ldots b_{n}\right]\right)=\left[f(a), f\left(b_{1}\right), \ldots, f\left(b_{n}\right)\right]$, for $i \leq n$. We show that $f\left(\left[a, b_{1}, \ldots, b_{n}, b_{(n+1)}\right]\right)=\left[f(a), f\left(b_{1}\right), \ldots, f\left(b_{n}\right), f\left(b_{n+1}\right)\right]$. For this, $\left[f(a), f\left(b_{1}\right), \ldots, f\left(b_{n}\right), f\left(b_{n+1}\right)\right]=\left[\left[f(a),\left(b_{1}\right), \ldots, f\left(b_{n}\right)\right], f\left(b_{n+1}\right)\right]=$ $\left[f\left(\left[a, b_{1}, \ldots, b_{n}\right]\right), f\left(b_{(n+1)}\right)\right]=f\left(\left[a, b_{1}, \ldots, b_{n+1}\right]\right)$. Thus, $f\left(\left[a, b_{1}, \ldots, b_{n+1}\right]\right)=$ $\left[f(a), f\left(b_{1}\right), \ldots, f\left(b_{n+1}\right)\right]$. Now, let $X$ be a nilpotent of class $n, y$ is an arbitrary elements of $Y$ and $y_{i} \in Y$, for $1 \leq i \leq n$. Then there exist $a, b_{i} \in X$, for $1 \leq i \leq n$ such that $f(a)=y$ and $f\left(b_{i}\right)=y_{i}$. Hence $0=f(0)=$ $f\left(\left[a, b_{1}, \ldots, b_{n}\right]\right)=\left[f(a), f\left(b_{1}\right), \ldots, f\left(b_{n}\right)\right]=\left[y, y_{1}, \ldots, y_{n}\right]$ that is, $Y$ is a nilpotent $B C I$-algebra. Conversely, let $Y$ be a nilpotent $B C I$-algebra of class $n$. Then for $a$ and any $b_{1}, \ldots, b_{n} \in X, f\left(\left[a, b_{1}, \ldots, b_{n}\right]\right)=\left[f(a), f\left(b_{1}\right), \ldots, f\left(b_{n}\right)\right]=$ $\left[y, y_{1}, \ldots, y_{n}\right]=0=f(0)$ and so $\left[a, b_{1}, \ldots, b_{n}\right]=0$, that is $X$ is a nilpotent $B C I$-algebra.

Corollary 4.5. If $X$ is nilpotent, then any subalgebra of $X$ is nilpotent, too. Also if $I$ is a $B C I$-ideal of $X$, then $X / I$ is nilpotent, too.

Lemma 4.6. $X / Z(X)$ is nilpotent of class $n$ if and only if $X$ is nilpotent of class $n+1$.

Proof. $\Longrightarrow$ Let $X / Z(X)$ be nilpotent of class $n$. Hence, $Z_{n}(X / Z(X))=$ $X / Z(X)$ and so for any $x \in X$ we have $\left[Z_{x}, Z_{y_{1}}, \ldots, Z_{y_{n}}\right]=Z_{0}$, for any $y_{1}, \ldots, y_{n} \in X$. Then by the proof of Theorem $3.14, Z_{\left[x, y_{1}, \ldots, y_{n}\right]}=Z_{0}$. Hence $\left[x, y_{1}, \ldots, y_{n}\right] \in Z(X)$ and so $\left[x, y_{1}, \ldots, y_{n}, y_{n+1}\right]=0$, for any $y_{n+1} \in X$, which implies that $x \in Z_{n+1}(X)$. Then, $Z_{n+1}(X)=X$. Therefore, $X$ is nilpotent of class $n+1$. 
$\Longleftarrow$ Let $X$ be a nilpotent $B C I$-algebra of class $n+1$. Then, by Corollary 4.5, $(f: X \longrightarrow X / Z(X)) X / Z(X)$ is nilpotent.

Theorem 4.7. The intersection of any two nilpotent subalgebras of $X$, is nilpotent.

Proof. The proof is straightforward.

Remak. By Theorem 4.2, every nilpotent $B C I$-algebra of class 1 , is a commutative $B C I$-algebra. Since commutative $B C I$-algebras form a variety, then every nilpotent $B C I$-algebras of class 1 form a variety.

Let $X$ be as in Example 3.13. Then for any $x, y, z \in X$ we have $[[x, y], z]=$ $[t, z]$ in which $t \in\{0,1,2\}$. Now since $N, N \cup A$ and $N \cup B$ are commutative $[[x, y], z]=[t, z]=0$. Therefore, $X$ is a nilpotent $B C I$-algebra.

The collection $\{N, A, B\}$ determines a partition of $X$. Thus we have a congruence relation $\theta$ on $X$ where $x \sim_{\theta} y$ if and only if both of $x$ and $y$ are in one of the tree sets $N, A$ and $B$. We obtain a quotient algebra $(X / \theta, *, N)$, where $X / \theta=\{N, A, B\}$. Now, it is easy to see that the "* " multiplication table on $X / \theta$ is

\begin{tabular}{l|lll}
$*$ & $\mathrm{~N}$ & $\mathrm{~A}$ & $\mathrm{~B}$ \\
\hline $\mathrm{N}$ & $\mathrm{N}$ & $\mathrm{N}$ & $\mathrm{N}$ \\
$\mathrm{A}$ & $\mathrm{A}$ & $\mathrm{N}$ & $\mathrm{N}$ \\
$\mathrm{B}$ & $\mathrm{B}$ & $\mathrm{N}$ & $\mathrm{N}$
\end{tabular}

Since $A * B=B * A=N$ and $A \neq B$, so $X / \theta$ is not a $B C I$-algebra. The mapping $f: X \longrightarrow X / \theta, x \longrightarrow \theta_{x}$ is an $B C I$-epimorphism. However, $X / \theta=f(X)$ is not a $B C I$-algebra. Thus, homomorphic image of an nilpotent $B C I$-algebra $X$ is not a nilpotent $B C I$-algebra.

Therefore, we have the following Theorem.

Theorem 4.8. Nilpotent BCI-algebras of class $n \neq 1$ do not form a variety.

Theorem 4.9. Let $I$ be an ideal of $X$ and $n, m \in \mathbb{N}$. If $I$ is a nilpotent $B C I$-ideal of class $m$ and $X / I$ is nilpotent of class $n$, then $X$ is nilpotent of class $(n+m)$.

Proof. The proof is similar to the proof of Theorem 3.14.

Theorem 4.10. Every BCI-algebra of order less than 5, is nilpotent.

Proof. The proof is similar to the proof of Theorem 3.15. 
Lemma 4.11. Let $X$ be a nilpotent $B C K(B C I)$-algebra of class $n \geq 1$ and $N$ be a nontrivial (closed) ideal of $X$. Then $N \cap Z(X) \neq 0$.

Proof. Since $X$ is nilpotent, so there exist $n \geq 1$ such that $Z_{n}(X)=X$. Thus $0=Z_{0}(X) \subseteq Z_{1}(X) \subseteq \ldots \subseteq Z_{n}(x)=X$. Since $N \cap Z_{n}(X)=N \cap X=$ $N \neq\{0\}$, there is $j \in \mathbb{N}$ such that $N \cap Z_{j}(X) \neq 0$. Let $i$ be the smallest index such that $N \cap Z_{i}(X) \neq 0$ ( so $N \cap Z_{i-1}(X)=0$ ). Then we claim that $\left[N \cap Z_{i}(X), X\right] \subseteq N$. For this let $w \in\left[N \cap Z_{i}(X), X\right]$. Then there exists $x \in N \cap Z_{i}(X)$ and $g \in X$ such that $w=[x, g]$. Since $x \in N(0 * x \in N)$ and $[x, g] \leq x,([x, g] *(0 * x) \leq x)$, then $w \in N$. Also since $w=[x, g]$ in which $x \in N \cap Z_{i}(X) \subseteq Z_{i}(X)$, by Lemma 4.3, $w \in Z_{i-1}(X)$. Hence $\left[N \cap Z_{i}(X), X\right] \subseteq N \cap Z_{i-1}(X)=0$. Then $N \cap Z_{i}(X) \leq Z(X)$ and so $N \cap Z_{i}(X) \leq N \cap Z(X)=0$. Hence $N \cap Z_{i}(X)=0$ which is a contradiction. Therefore, $N \cap Z(X) \neq 0$.

The following theorem shows that for a nilpotent $B C I(B C K)$-algebra $X$, each minimal ideal of $X$ is contained in $Z(X)$.

Theorem 4.12. Let $X$ be a nilpotent $B C K(B C I)$-algebra of class $n \geq 1$. If $N$ is a minimal (closed) ideal of $X$, then $N \leq Z(X)$.

Proof. Since $N$ and $Z(X)$ are ideals of $X$, we get $N \cap Z(X) \unlhd X$. Now since $N$ is a minimal ideal of $X, N \cap Z(X) \leq N$ and by Lemma $4.11,0 \neq N \cap Z(X)$. Hence, we get $N \cap Z(X)=N$. Therefore, $N \leq Z(X)$.

Theorem 4.13. Let $X$ be a nilpotent $B C K(B C I)$-algebra and $A$ be a maximal commutative (closed) ideal of $X$. Then

$$
A=C_{X}(A)=\langle\{x \in X \mid[x, a]=0,[a, x]=0 \text { for any } a \in A\}\rangle
$$

Proof. Clearly, $C_{X}(A)$ is an ideal of $X\left(C_{X}(A) \unlhd X\right)$. By Theorem 3.5, $A$ is 1-Engel and so $[x, y]=0$, for any $x, y \in A$, which implies that $A \subseteq C_{X}(A)$. Now, suppose $A \subsetneq C_{X}(A)$. Then $0 \neq \frac{C_{X}(A)}{A} \unlhd \frac{X}{A}$. Hence by Lemma 4.11, $\frac{C_{X}(A)}{A} \cap Z(\overline{X / A}) \neq 0$. So there exists $A_{0} \neq A_{g} \in \frac{C_{X}(A)}{A} \cap Z\left(\frac{X}{A}\right)$. Hence $g \in C_{X}(A)$ and so for $a \in A,[g, a]=0$ and $[a, g]=0$ which implies that $a *(a * g)=g *(g * a) \quad(*)$. Now let $B=\langle A, g\rangle$. Using $(*)$ and $A$ is commutative we see that $B$ is commutative too. Also $A \subsetneq B \unlhd X$. Therefore $B$ is a commutative ideal of $X$, which is a contradiction. Thus $A=C_{X}(A)$.

We recall that in ring theory, an element $x$ in $(R,+, \cdot)$ is a nilpotent element if $x^{n}=0$, for some positive integer $n$. Also, an ideal $I$ of $R$ is called nil ideal of $R$ if every element of $R$ is nilpotent. In [6], Huang used the notion of a nilpotent element of a ring to introduce the notion of a nilpotent element of 
a $B C I$-algebra. Moreover, the notions of nilpotent element, nilpotent $B C I$ algebra and nil ideal were introduced. In [13], [14] and [21], several results on these topics were obtained. In [13], for a non-empty subset $S$ of $B C I$-algebra $X$ and a positive integer $k, N_{k}(S)$ is defined to be $\left\{x \in S ; 0 * x^{n}=0\right\}$ and it is proved that for a closed ideal $A$ of $X$, the set of all nilpotent element of $A$ is a nil closed ideal of $A$. Also, $f^{-1}\left(N_{k}(A)\right)$ is an ideal of $X$ containing $N_{k}\left(f^{-1}(S)\right)$, where $f: X \rightarrow Y$ is a $B C I$ homomorphism and $A$ is an ideal of $Y$. In this section we used the notion of a nilpotent group to introduce a nilpotent $B C I$-algebra and in the following we see that there is no relation between nilpotent $B C I$-algebra in [6] an our definition in this paper.

Definition 4.14. [6] Let $X$ be a $B C I$-algebra and $x \in X$. If there exists a natural number $k$ such that $0 * x^{k}=0$, then $x$ is called a nilpotent element of $X$. An ideal $A$ of $X$ is called nil ideal of $X$ if every element of $A$ is nilpotent. Also, a $B C I$-algebra $X$ is called nilpotent, if every element in $X$ is nilpotent.

Theorem 4.15. [3] Every BCK-algebra is nilpotent. Every finite BCIalgebra is nilpotent.

Remark. Note that, $X$ in Example 3.9, is an infinite $B C K$-algebra which is not nilpotent, by our definition. Hence, by Theorem 4.15 , we can see that our definition is different of Definition 4.14. That is, if $X$ is nilpotent of class 1 , then $0 * x=0$ and so $X$ is a $B C K$-algebra. But in our definition, if $X$ is nilpotent of class 1 , then $X$ is a commutative $B C I$-algebra.

\section{Solvable $B C I$-algebras}

In [18] Najafi and et. all introduced solvable $B C I$-algebras by means of derived subgroup of $X$. In this section a new definition of derived subgroup of $X$ is introduced which help us to get more properties of solvable $B C I$-algebras.

Definition 5.1. Let $X$ be a $B C I$-algebra, $X^{(1)}=[X, X]=\langle\{[x, y]: x, y \in$ $X\}\rangle$ and for any $n \in \mathbb{N}, X^{(n)}=\left[X^{(n-1)}, X^{(n-1)}\right]$. Then $X$ is called solvable if there exists $n \in \mathbb{N}$ such that $X^{(n)}=0$. The smallest such $n$ is called derived lenght of $X$.

Theorem 5.2. [24] An ideal $A$ of a BCI-algebra $X$ is closed if and only if $0 * x \in A$, for any $x \in A$.

Theorem 5.3. Let $X$ be a BCI-algebra. Then $X^{(1)}$ is a 2-Engel closed ideal of $X$.

Proof. By Theorem 3.6, for any $[x, y] \in X^{(1)}, 0 *[x, y]=0 \in X^{(1)}$ which implies that $X^{(1)}$ is a closed ideal of $X$. Now, since for any $x, y \in X, 0 *[x, y]=0$, 
hence, $X^{(1)} \subseteq B$, in which $B$ is the $B C K$-part of $X$. Now, by Corollary 3.24, $X^{(1)}$ is 2-Engel.

Theorem 5.4. $X$ is commutative if and only if $X^{(1)}=0$.

Proof. $X$ is commutative if and only if for any $x, y \in x,[x, y]=0$ if and only if $X^{(1)}=0$.

Theorem 5.5. Let $I$ be an ideal of $X$. Then $X / I$ is commutative if and only if $X^{(1)} \subseteq I$.

Proof. By Theorem 3.4, X/I is a commutative if and only if , $\left[I_{x}, I_{y}\right]=I_{0}$, for any $x, y \in X$, if and only if for any $x, y \in X, I_{[x, y]}=I_{0}$ if and only if for any $x, y \in X,[x, y] \sim 0$ if and only if for all $x, y \in X,[x, y] * 0 \in I$ and $0 *[x, y] \in I$ (note that by Theorem 3.6, $0 *[x, y]=0$ ) if and only if for all $x, y \in X,[x, y] \in I$ if and only if $X^{(1)} \subseteq I$.

Theorem 5.6. Let $f$ be an isomorphism from $X$ to $B C I$-algebra $Y$ and $n \in \mathbb{N}$. Then $X$ is solvable if and only if $Y$ is solvable.

Proof. Let $f$ be an isomorphism from $X$ to $B C I$-algebra $Y$ and $x, y \in X$. Then $f([x, y])=[f(x), f(y)]$. Since $f(X)=Y$, we show that $f\left(X^{(1)}\right)=Y^{(1)}$. If $y \in f\left(X^{(1)}\right)$, then there exists $x \in X^{(1)}$ such that $f(x)=y$ and so $x \in X^{(1)}$, then there exist $a, b \in X$ such that $x=[a, b]$. Hence, $f(x)=f([a, b])=$ $f[a, b]=[f(a), f(b)]=y \in Y^{(1)}$. Then, $f\left(X^{(1)}\right) \subseteq Y^{(1)}$. Conversely, let $h \in Y^{(1)}$. Then, there exist $h_{1}, k \in Y$ such that $h=\left[h_{1}, k\right]$. However, $f$ is an isomorphism, hence there exist $a, b \in X$ such that $h_{1}=f(a)$ and $k=f(b)$. Thus, $h=\left[h_{1}, k\right]=[f(a), f(b)]=f[a, b] \in f\left(X^{(1)}\right)$. Then, we prove that $f\left(X^{(1)}\right)=Y^{(1)}$. Now, by induction on $n$, we can show that $f\left(X^{(n)}\right)=$ $Y^{(n)}$. Now, since $X$ is a solvable $B C I$-algebra, there exists $n \in \mathbb{N}$ such that $X^{(n)}=0$. Hence, $0=f(0)=f\left(X^{(n)}\right)=Y^{(n)}$ that is, $Y$ is a solvable $B C I$ algebra. Conversely, let $Y$ be a solvable $B C I$-algebra. Since $f(X)=Y$, we get $f\left(X^{(n)}\right)=Y^{(n)}=0$. Hence, $f\left(X^{(n)}\right)=0=f(0)$ and so $X^{(n)}=0$. Therefore, $X$ is a solvable $B C I$-algebra.

Corollary 5.7. If $X$ is solvable, then any subalgebra $X$ is solvable. Also if I is a $B C I$-ideal of $X$, then $X / I$ is solvable.

Theorem 5.8. The intersection of any two solvable subalgebras of $X$, is solvable.

Proof. The proof is straightforward. 
The above result can be generalized such that the intersection of any arbitrary family of subalgebras of an solvable $B C I$-algebra, is solvable.

Remark. Every solvable $B C I$-algebra of derived length $n=1$, is a commutative $B C I$-algebra. Since commutative $B C I$-algebras form a variety, then every solvable $B C I$-algebras derived length $n=1$ form a variety.

Let $X$ be as in Example 3.13. Then for any $x, y, z \in X$ we have $[[x, y],[z, t]]=$ $[t, u]$ in which $t, u \in\{0,1,2\}$. Now since $N$ is commutative $[[x, y],[z, t]]=$ $[t, u]=0$. Therefore, $X$ is a solvable $B C I$-algebra. Therefore, we have the following Theorem.

Theorem 5.9. Solvable BCI-algebras do not form a variety in general.

Theorem 5.10. Let $I$ be an ideal of $X$ and $n, m \in \mathbb{N}$. If $I$ and $X / I$ are solvable BCI-algebras, then $X$ is a solvable BCI-algebra.

Proof. Let $f$ be the natural homomorphism from $X$ onto $X / I$. Since $X / I$ is solvable, for some $n \in \mathbb{N}, f\left(X^{(n)}\right)=(X / I)^{(n)}=I$. Hence $X^{(n)}$ is a subalgebra of $\operatorname{ker}(f)=I$. By Corollary 5.7, $X^{(n)}$ is solvable. Hence, there exists a positive integer number $k$ such that $X^{(n+k)}=\left(X^{(n)}\right)^{(k)}=0$. That is $X$ is solvable.

Theorem 5.11. If $X$ is finite and $[x, y] \leq x$, for any $x, y \in X$, then it is solvable.

Proof. Let $X$ be finite, $|X|=n \geq 2$ and $M$ be the set of all its maximal elements. Then $M$ has at least one element and $[0, y]=0 \notin M$ for every $y \in X$. For $x \neq 0$ there is $m \in M$ such that $x \leq m$. By hypotheses, $[x, y]<x \leq m$, so $[x, y] \notin M$ for all $x, y \in X$. Also by Theorem $5.3, X^{(1)}$ is a subalgebra contained in $X \backslash M$. Hence $|X|>\left|X^{(1)}\right|$. By induction $|X|>$ $\left|X^{(1)}\right|>\left|X^{(2)}\right|>>\left|X^{(k)}\right|=1$, for some $k<n$. It is not difficult to see that $k$ is less than the length of the longest sequence $0<a_{1}<a_{2}<<a_{m}$ of elements of X. Thus, for some $k<n,\left|X^{(k)}\right|=1$ which implies that $X^{(k)}=\{0\}$. Hence, $X$ is solvable.

Corollary 5.12. Every finite BCK-algebra is solvable.

Proof. It is clear by Lemma 2.4 and Theorem 5.11.

In the following Example we have an infinite $B C K$-algebra which is not solvable.

Example 5.13. Let $X=[0,1]$ and " *" is given by

$$
x * y=\left\{\begin{array}{lc}
0, & x \leq y \\
x, & \text { otherwise }
\end{array}\right.
$$


Then $(X, *, 0)$ is an infinite BCK-algebra and $X^{(n)}=[0,1)$ for any $n \geq 1$. Then $X$ is not solvable.

\section{Relation between Engel, nilpotent and solvable $B C I$ - algebras}

Here, we can justify our definition of Engel, nilpotent and solvable $B C I$ algebras. In group theory each nilpotent group is Engel and solvable. This initial study can verify, as we have done in Theorems 6.1 and 6.3 , that each nilpotent $B C I$-algebras is Engel and solvable.

Theorem 6.1. If $X$ is a nilpotent BCI-algebra, then $X$ is an Engel.

Proof. Let $X$ be nilpotent. Then there exist $n \in \mathbb{N}$ such that $Z_{n}(X)=X$. Hence, for any $x, y \in X$, we have $[x, \underbrace{y, \ldots y}_{n-\text { times }}]=0$, which implies that $X$ is an $n$-Engel.

Remark The converse of Theorem 6.1, is not true in general. In the following Example we have a 2-Engel BCI- algebra which is not nilpotent.

Example 6.2. Let $X=[0,1]$ and operation " *" be given by:

$$
x * y=\left\{\begin{array}{lc}
0, & x \leq y \\
x, & \text { otherwise }
\end{array}\right.
$$

By Example 3.9, $X$ is a 2-Engel BCK-algebra but $X$ is not nilpotent. For this let $y_{3} \leq y_{2} \leq y_{1} \leq x$. Then $\left[x, y_{1}\right]=y 1$ and so $\left[y_{1}, y_{2}\right]=y_{2}$ which implies that $\left[x, y_{1}, y_{2}, y_{3}\right]=\left[y_{1}, y_{2}, y_{3}\right]=\left[y_{2}, y_{3}\right]=y_{3}$. Repeating this we see that there is not $n \geq 0$ such that $\left[x, y_{1}, y_{2}, \ldots, y_{n}\right]=0$. Hence, $X$ is a 2-Engel $B C K$-algebra which is not nilpotent.

Now, let $Z$ be as in Example 3.9, then $Z$ is a 2-Engel BCI-algebra. Now, we show that $Z$ is a 2-Engel BCI-algebra that is not nilpotent. By the same manipulation of Example 3.9, we have

$$
\left[\left(x_{1}, y_{1}\right),\left(x_{2}, y_{2}\right), \ldots,\left(x_{n}, y_{n}\right)\right]=\left(\left[x_{1}, x_{2}, \ldots, x_{n}\right],\left[y_{1}, y_{2}, \ldots, y_{n}\right]\right)
$$

for any $x_{1}, \ldots, x_{n} \in X$ and $y_{1}, \ldots, y_{n} \in Y$. Since $X$ is not nilpotent, so there is not $n \geq 0$ such that $\left[x_{1}, x_{2}, \ldots, x_{n}\right]=0$, where $x_{1} \geq x_{2} \geq \ldots \geq x_{n}$. Then, $\left[\left(x_{1}, y_{1}\right),\left(x_{2}, y_{2}\right), \ldots,\left(x_{n}, y_{n}\right)\right] \neq(0,0)$ which implies that there is not $n \in \mathbb{N}$ such that $Z_{n}(Z)=Z$ and so $Z$ is not a nilpotent BCI-algebra.

Theorem 6.3. Let $X$ be a finite nilpotent BCI-algebra. Then $X$ is solvable. 
Proof. Let $X$ be a finite nilpotent $B C I$-algebra of order $n$. We do the proof by induction on $n$. Since $X$ is nilpotent so there is $m \in \mathbb{N}$ such that $Z_{m}(X)=X$. Now, consider $Y=\frac{X}{Z(X)}$. Using Theorem 4.6, $Y$ is a nilpotent $B C I$-algebra of order $t<n$ and so by hypotheses of induction $Y$ is solvable. Also $Z(X)$ is a commutative $B C I$-algebra and so $Z(X)$ is solvabel. Therefore, by Theorem $5.10, X$ is solvable.

In the following Example we have a finite nilpotent $B C I$-algebra which is solvable.

Example 6.4. Let $X=\{0,1, \ldots, n\}$ and operation " * be given by:

$$
x * y=\left\{\begin{array}{lc}
0, & x \leq y \\
x, & \text { otherwise }
\end{array}\right.
$$

Clearly, $X$ is a nilpotent BCK-algebra and by Corollary 5.12, $X$ is solvable. Now, if $Z$ denote the cartesian product $X \times Y$ where $Y$ is as in Example 3.2. Then $Z$ is a BCI-algebra, where operation "*" is defined by $\left(x_{1}, y_{1}\right) *\left(x_{2}, y_{2}\right)=$ $\left(x_{1} * y_{1}, x_{2} * y_{2}\right)$ for any $x_{1}, x_{2} \in X$ and $y_{1}, y_{2} \in Y$ (See [24]). Since $Y$ is a commutative BCI-algebra so $Y$ is a nilpotent and solvable BCI-algebra. Therefore, $Z$ is a solvable and nilpotent BCI-algebra.

In the following we show that the notion of 1-Engel $B C I$-algebras and quasi-commutative $B C I$-algebras are not equivalent.

Definition 6.5. [3] A BCI-algebra $X$ is called quasi-commutative of type $(i, j, m, n)$, if there exist two pairs of non-negative integers $i, j$ and $m, n$ such that the following identity holds:

$$
\left(x *(x * y)^{i+1}\right) *(y * x)^{j}=\left(y *(y * x)^{m+1}\right) *(x * y)^{n}
$$

Remark. Every 1-Engel $B C I$-algebra is a commutative $B C I$-algebra. Also, every quasi-commutative $B C I$-algebra is not a commutative $B C I$-algebra (See [3]). Therefore, the notion of 1-Engel $B C I$-algebras and quasi-commutative $B C I$-algebras are not equivalent. By the same method the notion of nilpotent (solvable) $B C I$-algebras and quasi-commutative $B C I$-algebras are not equivalent.

In the following example we have an 1-Engel $B C I$-algebra which is not a quasi-commutative $B C I$-algebra.

Example 6.6. Assume that $(N, *, 0)$ is the commutative BCK-chain in which $x * y=\max \{0, x-y\}$ and let $(P, *, 0)$ be a nonzero $p$-semisimple algebra. Then $N$ and $P$ are two commutative BCI-algebras. So $N \times P$ is an 1-Engel BCIalgebra. But $N \times P$ can not be quasi-commutative (See [3]). 


\section{Conclusions}

By the notion of Engel (nilpotent, solvable) $B C I(B C K)$-algebra we find the necessary and sufficient condition under which each special commutator of weight $n \geq 1$ is equal to 0 . Therefore, we think it could be useful to investigate on condition under which all $B C I(B C K)$-chains of a given $B C I(B C K)$ algebra terminate at finite step.

\section{Acknowledgments}

The authors wish to sincerely thank the referee for several useful comments.

\section{References}

[1] R. Ameri, R. A. Borzooei, E. Mohammadzadeh, Engel fuzzy subgroups, Italian Journal of Pure and Applied Mathematics, 34 (2015), 251- 262.

[2] R. A. Borzooei, E. Mohammadzadeh, Violeta Fotea, On Engel Fuzzy Subpolygroups, New Mathematics and Natural Computation, 13 (2) (2017), $195-206$.

[3] B. Davvaz, Polygroup theory and related systems , World Scientific, 2013.

[4] E. S. Golod, Some problems of Burnside type, in Proc. Int. Congr. Math., Moscow, (1966), 284-289.

[5] H. Heineken, Engelsche Elemente der Lange drei, Illionis J. Math., 5 ( 1961), 681-707.

[6] W. Hung, Nil-radical in BCI-algebras, Math. Japon, 37 (1992), 363-366.

[7] Y. Imai, K. Iseki, On axiom systems of propositional calculi XIV, Proc. Japan Academy, 42 (1966), 19-22.

[8] K. Iseki, An algebras related with a propositional calculus, Math. Japonica, 42 (1966), 26-29.

[9] K. Iseki, BCK-algebras, Math. Seminar Notes, 4 (1976), 77-86.

[10] K. Iseki, On ideals in BCK-algebras, Math. Seminar Notes, 3 (1975), $1-12$.

[11] K. Iseki, On BCI-algebras, Math. Sem Notes, 8 (1980), 125-130. 
[12] K. Iseki, S. Tanaka, A. Rosenfeld, An introduction to theory of BCKalgebras, Math. Japonica, 23 (1978), 1-26.

[13] Y. B. Jun and E. H. Roh, Nil ideals in BCI-algebras, Math. Japon., 41(2) (1995), 297-302.

[14] Y. B. Jun, J. Meng and E. H. Roh, On nil ideals in BCI-algebras, Math. Japon., 38 (1993), 1051-1056.

[15] L. C. Kappe, W. P. Kappe, On three-Engle groups, Bull. Austral. Math. Soc., 7 (1972), 391-405.

[16] J. Meng, Y. B. Jun, BCK-algebras, Kyung Moon Sa Co. Seoul, Korea, (1994).

[17] E. Mohammadzadeh, R. A. Borzooei, Nilpotent Fuzzy Subgroups, Mathematics, 6, 27(2018); doi:10.3390/math 60200027.

[18] A. Najafi, Peseudo-commutators in BCK algebras, Pure Mathematical Science, 2(1) (2013), 29-32.

[19] A. Najafi, A. B. Saeid, Solvable BCK algebras, Cankaya University Journal of Science and Engineering, 11(2) (2014), 19-28.

[20] A. Najafi, A. Borumand Saeid, E. Elami, Commutators in BCI algebras, Journal of Intelligent and Fuzzy Systems, 31 (2016), 357-366.

[21] X. Pin, The study of nilpotent element in BCI-algebras, Journal of Shaanxi University of Technology, 30 (2003), 112-123.

[22] D. J. S. Robinson, A Course in the theory of groups, Springer-Verlag (1980).

[23] A. Wronski, BCK-algebras do not form a variety, Math. Japonica, 28 (1983), 211-213.

[24] H. Yisheng, BCI Algebra, Science Pess, 2003. 